\title{
Determinants of multiplicative Toeplitz matrices
}

\author{
by
}

Titus Hilberdink (Reading)

Introduction. In this article, we consider matrices of the form

$$
\left(\begin{array}{ccccc}
c(1) & c(1 / 2) & c(1 / 3) & c(1 / 4) & \cdots \\
c(2) & c(1) & c(2 / 3) & c(1 / 2) & \cdots \\
c(3) & c(3 / 2) & c(1) & c(3 / 4) & \cdots \\
c(4) & c(2) & c(4 / 3) & c(1) & \cdots \\
\vdots & \vdots & \vdots & \vdots & \ddots
\end{array}\right)
$$

and their determinants, where the $(i, j)$ th entry is a function of $i / j$. They are characterised by being constant along lines where $i / j$ is a given positive rational. In this sense they resemble Toeplitz matrices, which are constant on lines parallel to the diagonal, i.e. where $i-j$ is a given integer. For this reason we shall call them multiplicative Toeplitz matrices. These matrices represent linear operators between various spaces. To identify these spaces, and to determine when they are bounded, are interesting questions in themselves, but for the purposes of this paper, these will not concern us. We shall be mainly concerned with determinants of truncated matrices.

Toeplitz matrices are most usefully studied by associating them with a function (or "symbol") whose Fourier coefficients make up the matrix. Indeed, they generate bounded operators on $\ell^{2}$ if and only if the matrix entries are the Fourier coefficients of an essentially bounded function on $\mathbb{T}$ (the unit circle). To matrices of the form (0.1), we associate, by analogy, the series

$$
\sum_{q \in \mathbb{Q}^{+}} c(q) q^{i t},
$$

where $q$ ranges over the positive rationals. We shall first make sense of such series in $\S 1$.

2000 Mathematics Subject Classification: Primary 11C20; Secondary 15A15, 15A23, $65 \mathrm{~F} 40$. 
In $\S 2$, we concern ourselves with the case when $c(\cdot)$ is a multiplicative function on the positive rationals. Then the matrix (0.1) and the series (0.2) are shown to factorise as "Euler products".

In $\S 3$, we consider the determinants of truncated multiplicative Toeplitz matrices for which $c(\cdot)$ is multiplicative. The "Euler product" formula ceases to hold when we truncate the matrices, but we show in Theorem 3.2 that it is miraculously recovered on taking determinants. This reduces the problem of evaluating such determinants to those whose non-zero entries lie on "lines" $i / j=p^{k}$ for $p$ prime and $k \in \mathbb{Z}$. It is shown in Theorem 3.1 that these can be evaluated in terms of determinants of Toeplitz matrices. The proofs are done separately in $\S 4$. Using this formula, and known formulae for Toeplitz determinants with rational symbol, we evaluate several determinants of number-theoretic interest in $\S 5$. For example, for $f$ and $g$ completely multiplicative,

$$
\operatorname{det}\left(f\left(\frac{i}{(i, j)}\right) g\left(\frac{j}{(i, j)}\right)\right)_{i, j \leq n}=\prod_{p \leq n}(1-f(p) g(p))^{[n / p]} .
$$

Determinants associated with arithmetical functions have been studied and evaluated by many authors. A number of generalisations have been made of Smith's determinant [7] of the matrix with coefficients $(i, j)$ (see for example [6]). The matrices considered are however of a different form; e.g. in [6], the first column is constant. Generalisations in a different direction were made in [2], [5], and also [3], but again the matrices considered are of a different form from $(0.1)$.

1. $\mathbb{Q}^{+}$-coefficients and series. For a function $c: \mathbb{Q}^{+} \rightarrow \mathbb{C}$ defined on the positive rationals, we define

$$
\sum_{q \in \mathbb{Q}^{+}} c(q)=\lim _{N \rightarrow \infty} \sum_{\substack{m, n \leq N \\(m, n)=1}} c(m / n), \quad \text { whenever this limit exists. }
$$

We sometimes abbreviate the left-hand sum by $\sum_{q} c(q)$. For $q=m / n \in \mathbb{Q}^{+}$ with $(m, n)=1$, we write $|q|=\max \{m, n\}$. Thus the above definition becomes

$$
\sum_{q} c(q)=\lim _{N \rightarrow \infty} \sum_{|q| \leq N} c(q) .
$$

Note that if $f(t)=\sum_{q \in \mathbb{Q}^{+}} c(q) q^{i t}$ and the sum converges absolutely, then for $x>0$,

$$
\begin{aligned}
\lim _{T \rightarrow \infty} \frac{1}{2 T} \int_{-T}^{T} f(t) x^{-i t} d t & =\lim _{T \rightarrow \infty} \frac{1}{2 T} \int_{-T}^{T} \sum_{q \in \mathbb{Q}^{+}} c(q) q^{i t} x^{-i t} d t \\
& =\sum_{q \in \mathbb{Q}^{+}} c(q) \lim _{T \rightarrow \infty} \frac{1}{2 T} \int_{-T}^{T}(q / x)^{i t} d t= \begin{cases}c(x) & \text { if } x \in \mathbb{Q}^{+}, \\
0 & \text { if } x \notin \mathbb{Q}^{+} .\end{cases}
\end{aligned}
$$


More generally, given a measurable function $f: \mathbb{R} \rightarrow \mathbb{C}$, we can ask if the limit

$$
\lim _{T \rightarrow \infty} \frac{1}{2 T} \int_{-T}^{T} f(t) x^{-i t} d t
$$

exists for every $x>0$. If it exists, we shall denote it by $c(x)$ or, if we want to emphasise the dependence on $f$, by $c_{f}(x)$. This limit is also called the "Hadamard coefficient" (see [8], [4]). Of particular number-theoretic interest are those $f$ for which $c(x)$ is supported on the positive rationals; that is,

$$
c(x)=0 \quad \text { for } x \notin \mathbb{Q}^{+} .
$$

DEFINITION 1.1.

(a) Let $V$ denote the space of measurable functions $f$ for which (1.1) and (1.2) hold. We call $c(q)$ (for $q \in \mathbb{Q}^{+}$) the $\mathbb{Q}^{+}$-coefficients of $f$ and write

$$
f(t) \sim \sum_{q \in \mathbb{Q}^{+}} c(q) q^{i t}
$$

which we call the $\mathbb{Q}^{+}$-series of $f$. (The series formed as such, is a formal series and need not converge.)

(b) Let $U$ denote the subspace of $V$ consisting of all absolutely convergent $\mathbb{Q}^{+}$-series; that is,

$$
U=\left\{\sum_{q \in \mathbb{Q}^{+}} c(q) q^{i t}: \sum_{q \in \mathbb{Q}^{+}}|c(q)|<\infty\right\} .
$$

(c) For $f \in V$, with $\mathbb{Q}^{+}$-coefficients $c(q)$, let $A(f)$ denote the infinite matrix

$$
A(f)=(c(i / j))_{i, j \geq 1} .
$$

Proposition 1.2 Let $f, g \in U$. Then $f g \in U$ and

$$
c_{f g}(q)=\sum_{r \in \mathbb{Q}^{+}} c_{f}(r) c_{g}(q / r) .
$$

Proof. If $f(t)=\sum_{r \in \mathbb{Q}^{+}} c_{f}(r) r^{i t}$ and $g(t)=\sum_{s \in \mathbb{Q}^{+}} c_{g}(s) s^{i t}$ we have, by the absolute convergence of the series,

$$
f(t) g(t)=\sum_{r, s \in \mathbb{Q}^{+}} c_{f}(r) c_{g}(s)(r s)^{i t}=\sum_{q \in \mathbb{Q}^{+}} q^{i t}\left(\sum_{r \in \mathbb{Q}^{+}} c_{f}(r) c_{g}(q / r)\right),
$$

from which the result follows.

Examples 1.3. (a) Let $g(s)=\sum_{n=1}^{\infty} a_{n} / n^{s}$ and $h(s)=\sum_{n=1}^{\infty} b_{n} / n^{s}$ be two Dirichlet series which converge absolutely for $\Re s>\sigma_{0}$. Let $\alpha, \beta>\sigma_{0}$ 
and put $f(t)=g(\alpha-i t) h(\beta+i t)$. Then $f \in U$ with

$$
c(q)=\frac{1}{m^{\alpha} n^{\beta}} \sum_{d=1}^{\infty} \frac{a_{m d} b_{n d}}{d^{\alpha+\beta}} \quad \text { for } q=\frac{m}{n} \text { with }(m, n)=1 .
$$

We can prove this by multiplying out the series for $g(\alpha-i t)$ and $h(\beta+i t)$. We have

$$
\begin{aligned}
f(t) & =\sum_{m=1}^{\infty} \frac{a_{m}}{m^{\alpha-i t}} \sum_{n=1}^{\infty} \frac{b_{n}}{n^{\beta+i t}}=\sum_{m, n \geq 1} \frac{a_{m} b_{n}}{m^{\alpha} n^{\beta}}\left(\frac{m}{n}\right)^{i t} \\
& =\sum_{d=1}^{\infty} \sum_{\substack{m, n \geq 1 \\
(m, n)=d}} \frac{a_{m} b_{n}}{m^{\alpha} n^{\beta}}\left(\frac{m}{n}\right)^{i t}=\sum_{d=1}^{\infty} \frac{1}{d^{\alpha+\beta}} \sum_{\substack{m, n \geq 1 \\
(m, n)=1}} \frac{a_{m d} b_{n d}}{m^{\alpha} n^{\beta}}\left(\frac{m}{n}\right)^{i t} \\
& =\sum_{\substack{m, n \geq 1 \\
(m, n)=1}} \frac{1}{m^{\alpha} n^{\beta}}\left(\sum_{d=1}^{\infty} \frac{a_{m d} b_{n d}}{d^{\alpha+\beta}}\right)\left(\frac{m}{n}\right)^{i t} .
\end{aligned}
$$

(b) As a special case of the above, take $a_{n}=b_{n}=1$ for all $n$. Then, for $\alpha, \beta>1$, we have

$$
\frac{\zeta(\alpha-i t) \zeta(\beta+i t)}{\zeta(\alpha+\beta)}=\sum_{q \in \mathbb{Q}^{+}} c(q) q^{i t}=\sum_{\substack{m, n \geq 1 \\(m, n)=1}} \frac{1}{m^{\alpha} n^{\beta}}\left(\frac{m}{n}\right)^{i t},
$$

where $\zeta(\cdot)$ is the Riemann zeta function. Note that this gives, for arbitrary $m, n \in \mathbb{N}$,

$$
c\left(\frac{m}{n}\right)=c\left(\frac{m /(m, n)}{n /(m, n)}\right)=\frac{(m, n)^{\alpha+\beta}}{m^{\alpha} n^{\beta}} .
$$

2. Multiplicative coefficients and Euler products. We start by extending the notion of a multiplicative function to functions defined on the positive rationals. Recall that a function $f: \mathbb{N} \rightarrow \mathbb{C}$ is multiplicative if it is not identically zero and $f(m n)=f(m) f(n)$ whenever $(m, n)=1$. This is equivalent to saying that $f$ satisfies: $f(1)=1$ and

$$
f\left(p_{1}^{a_{1}} \cdots p_{k}^{a_{k}}\right)=f\left(p_{1}^{a_{1}}\right) \cdots f\left(p_{k}^{a_{k}}\right)
$$

for all distinct primes $p_{i}$ and all $a_{i} \in \mathbb{N}$.

Definition 2.1. A function $a: \mathbb{Q}^{+} \rightarrow \mathbb{C}$ is multiplicative if $a(1)=1$ and

$$
a\left(p_{1}^{a_{1}} \cdots p_{k}^{a_{k}}\right)=a\left(p_{1}^{a_{1}}\right) \cdots a\left(p_{k}^{a_{k}}\right)
$$

for all distinct primes $p_{i}$ and all $a_{i} \in \mathbb{Z} \backslash\{0\}$. 
We say $a$ is completely multiplicative if, in addition to the above, we also have, for all primes $p$ and $k \in \mathbb{N}$,

$$
a\left(p^{k}\right)=a(p)^{k} \quad \text { and } \quad a\left(p^{-k}\right)=a\left(p^{-1}\right)^{k} .
$$

The next proposition follows immediately from the definition.

Proposition 2.2. Let $a: \mathbb{Q}^{+} \rightarrow \mathbb{C}$. Then $a$ is multiplicative if and only if there exist multiplicative functions $f, g: \mathbb{N} \rightarrow \mathbb{C}$ such that $a(m / n)=$ $f(m) g(n)$ whenever $(m, n)=1$. Furthermore, a is completely multiplicative if and only if both $f$ and $g$ are.

Next we show that coefficients derived from Dirichlet series with multiplicative coefficients as in example (a) are also multiplicative.

TheOREm 2.3. Let $a, b: \mathbb{N} \rightarrow \mathbb{C}$ be multiplicative functions such that $\sum_{d=1}^{\infty} a(m d) b(n d)$ converges absolutely for every $m, n \geq 1$ and its sum is non-zero if $m=n=1$. Then the function $c: \mathbb{Q}^{+} \rightarrow \mathbb{C}$ is multiplicative, where $c(\cdot)$ is defined by

$$
c\left(\frac{m}{n}\right)=\frac{\sum_{d=1}^{\infty} a(m d) b(n d)}{\sum_{d=1}^{\infty} a(d) b(d)} \quad \text { for }(m, n)=1 .
$$

Proof. First $c(1)=1$. Now consider $(m, n)=1$. Write $m=\prod_{p} p^{\alpha}$ and $n=\prod_{p} p^{\beta}$. Note that $\alpha=0$ for $p \mid n$, while $\beta=0$ for $p \mid m$. Since $a$ and $b$ are multiplicative we have

$$
\begin{aligned}
c\left(\frac{m}{n}\right) \sum_{d=1}^{\infty} a(d) b(d) & =\prod_{p} \sum_{k=0}^{\infty} a\left(p^{k+\alpha}\right) b\left(p^{k+\beta}\right) \\
& =\prod_{p \nmid m n} \sum_{k=0}^{\infty} a\left(p^{k}\right) b\left(p^{k}\right) \prod_{p \mid m n} \sum_{k=0}^{\infty} a\left(p^{k+\alpha}\right) b\left(p^{k+\beta}\right) \\
& =\prod_{p} \sum_{k=0}^{\infty} a\left(p^{k}\right) b\left(p^{k}\right) \prod_{p \mid m n} \frac{\sum_{k=0}^{\infty} a\left(p^{k+\alpha}\right) b\left(p^{k+\beta}\right)}{\sum_{k=0}^{\infty} a\left(p^{k}\right) b\left(p^{k}\right)} \\
& =\sum_{d=1}^{\infty} a(d) b(d) \prod_{p \mid m n} \frac{\sum_{k=0}^{\infty} a\left(p^{k+\alpha}\right) b\left(p^{k+\beta}\right)}{\sum_{k=0}^{\infty} a\left(p^{k}\right) b\left(p^{k}\right)} .
\end{aligned}
$$

(Note that here we used the fact that $\sum_{k=0}^{\infty} a\left(p^{k}\right) b\left(p^{k}\right) \neq 0$, which follows from $\sum_{n=1}^{\infty} a(n) b(n) \neq 0$.) Hence

$$
\begin{aligned}
c\left(\frac{m}{n}\right) & =\prod_{p \mid m} \frac{\sum_{k=0}^{\infty} a\left(p^{k+\alpha}\right) b\left(p^{k}\right)}{\sum_{k=0}^{\infty} a\left(p^{k}\right) b\left(p^{k}\right)} \prod_{p \mid n} \frac{\sum_{k=0}^{\infty} a\left(p^{k}\right) b\left(p^{k+\beta}\right)}{\sum_{k=0}^{\infty} a\left(p^{k}\right) b\left(p^{k}\right)} \\
& =f(m) g(n),
\end{aligned}
$$


where

$$
f(m)=\prod_{p \mid m} \frac{\sum_{k=0}^{\infty} a\left(p^{k+\alpha}\right) b\left(p^{k}\right)}{\sum_{k=0}^{\infty} a\left(p^{k}\right) b\left(p^{k}\right)}, \quad g(n)=\prod_{p \mid n} \frac{\sum_{k=0}^{\infty} a\left(p^{k}\right) b\left(p^{k+\beta}\right)}{\sum_{k=0}^{\infty} a\left(p^{k}\right) b\left(p^{k}\right)} .
$$

After Proposition 2.2, it remains to show that $f$ and $g$ are multiplicative.

Consider $f$. Again, let $(m, n)=1$ and write $m=\prod_{p} p^{\alpha}$ and $n=\prod_{p} p^{\beta}$. Then

$$
\begin{aligned}
f(m n) & =\prod_{p \mid m n} \frac{\sum_{k=0}^{\infty} a\left(p^{k+\alpha+\beta}\right) b\left(p^{k}\right)}{\sum_{k=0}^{\infty} a\left(p^{k}\right) b\left(p^{k}\right)} \\
& =\prod_{p \mid m} \frac{\sum_{k=0}^{\infty} a\left(p^{k+\alpha}\right) b\left(p^{k}\right)}{\sum_{k=0}^{\infty} a\left(p^{k}\right) b\left(p^{k}\right)} \prod_{p \mid n} \frac{\sum_{k=0}^{\infty} a\left(p^{k+\beta}\right) b\left(p^{k}\right)}{\sum_{k=0}^{\infty} a\left(p^{k}\right) b\left(p^{k}\right)}=f(m) f(n),
\end{aligned}
$$

showing $f$ is multiplicative. Similarly, $g$ is multiplicative.

Euler products. Dirichlet series with multiplicative coefficients can be factorised as Euler products. We show below that this factorisation extends naturally to $\mathbb{Q}^{+}$-series with multiplicative coefficients and, furthermore, to the corresponding multiplicative Toeplitz matrix.

Suppose $f \in U$ and $f$ has multiplicative $\mathbb{Q}^{+}$-coefficients $c(q)$. For a prime $p$ and $t$ real, we define $f_{p}(t)$ by

$$
f_{p}(t)=\sum_{k \in \mathbb{Z}} c\left(p^{k}\right) p^{i k t}
$$

Let $n$ be a squarefree number greater than 1 . We define $f_{n}(t)$ by

$$
f_{n}(t)=\prod_{p \mid n} f_{p}(t)
$$

Let $S_{n}$ denote the set of all rationals formed from the prime divisors of $n$, i.e. if $n=p_{1} \cdots p_{k}$ then

$$
S_{n}=\left\{p_{1}^{a_{1}} \cdots p_{k}^{a_{k}}: a_{1}, \ldots, a_{k} \in \mathbb{Z}\right\} .
$$

By multiplicativity of $c(\cdot)$ and the fundamental theorem of arithmetic, it follows that

$$
f_{n}(t)=\sum_{q \in S_{n}} c(q) q^{i t} .
$$

Theorem 2.4. Let $f \in U$ whose $\mathbb{Q}^{+}$-coefficients $c(q)$ are multiplicative. Then we have the Euler products

(i) $f(t)=\prod_{p} f_{p}(t)$,

(ii) $A(f)=\prod_{p} A\left(f_{p}\right)$. 
Proof. (i) From above we have

$$
\prod_{p \leq P} f_{p}(t)=\sum_{q \in S_{Q}} c(q) q^{i t}
$$

where $Q=\prod_{p \leq P} p$. Note that every $q$ with $|q| \leq P$ is in $S_{Q}$. Hence

$$
\begin{aligned}
\left|f(t)-\prod_{p \leq P} f_{p}(t)\right| & =\left|\sum_{q \in \mathbb{Q}^{+}} c(q) q^{i t}-\sum_{q \in S_{Q}} c(q) q^{i t}\right| \\
& \leq \sum_{|q|>P}|c(q)| \rightarrow 0 \quad \text { as } P \rightarrow \infty,
\end{aligned}
$$

from which the result follows.

(ii) First we prove that for $m, n$ coprime,

$$
A\left(f_{m} f_{n}\right)=A\left(f_{m}\right) A\left(f_{n}\right) .
$$

By Proposition 1.2, $f_{m}, f_{n} \in U$ implies $f_{m} f_{n} \in U$, with

$$
c_{f_{m} f_{n}}(q)=\sum_{r \in \mathbb{Q}^{+}} c_{f_{m}}(r) c_{f_{n}}(q / r) .
$$

Replacing $r$ with $i / q$ gives

$$
A\left(f_{m} f_{n}\right)_{i j}=c_{f_{m} f_{n}}(i / j)=\sum_{q \in \mathbb{Q}^{+}} c_{f_{m}}(i / q) c_{f_{n}}(q / j) .
$$

On the other hand,

$$
\left(A\left(f_{m}\right) A\left(f_{n}\right)\right)_{i j}=\sum_{r=1}^{\infty} c_{f_{m}}(i / r) c_{f_{n}}(r / j) .
$$

Hence we must show that there is no contribution in (2.3) if $q \notin \mathbb{N}$.

We can write $i=s_{1} t_{1} u$ and $j=s_{2} t_{2} v$, where $s_{1}, s_{2} \in S_{m}, t_{1}, t_{2} \in S_{n}$ and $u, v$ are coprime to $m n$. Since $i, j \in \mathbb{N}$, this forces $s_{1}, s_{2}, t_{1}, t_{2}, u, v \in \mathbb{N}$. For a contribution to (2.3), we need $i / q \in S_{m}$ and $q / j \in S_{n}$. Hence

$$
q=i s=j t \quad \text { for some } s \in S_{m} \text { and } t \in S_{n} .
$$

But then $s_{1} s t_{1} u=s_{2} t_{2} t v$, which forces $s_{1} s=s_{2}, t_{1}=t_{2} t$, and $u=v$. As a result, $q=i s_{2} / s_{1}=s_{2} t_{1} u \in \mathbb{N}$.

Applying (2.2) repeatedly we have, for every $P$,

$$
A\left(\prod_{p \leq P} f_{p}\right)=\prod_{p \leq P} A\left(f_{p}\right) .
$$

Fix $i, j$, and let $P \geq \max \{i, j\}$. Then the matrices $A\left(f_{p}\right)$ for $p>P$ do not affect the $(i, j)$ th entry on the right. Similarly, on the left, the $(i, j)$ th entry 
equals that of $A(f)$. Thus

$$
A(f)=\prod_{p} A\left(f_{p}\right)
$$

3. Determinants. In this section we obtain a formula for the determinants of truncated multiplicative Toeplitz matrices in the case where the $\mathbb{Q}^{+}$-coefficients are multiplicative. Let $f \in U$ with multiplicative $\mathbb{Q}^{+}$coefficients $c(q)$. We denote by $A_{N}(f)$ the truncated $N \times N$-matrix for $A(f)$; that is,

$$
A_{N}(f)=\left(\begin{array}{cccccc}
c(1) & c(1 / 2) & c(1 / 3) & c(1 / 4) & \cdots & c(1 / N) \\
c(2) & c(1) & c(2 / 3) & c(1 / 2) & \cdots & c(2 / N) \\
c(3) & c(3 / 2) & c(1) & c(3 / 4) & \cdots & c(3 / N) \\
c(4) & c(2) & c(4 / 3) & c(1) & \cdots & c(4 / N) \\
\vdots & \vdots & \vdots & \vdots & \ddots & \vdots \\
c(N) & c(N / 2) & c(N / 3) & c(N / 4) & \cdots & c(1)
\end{array}\right) .
$$

From Theorem 2.4 we have the Euler product formula

$$
A(f)=\prod_{p} A\left(f_{p}\right) .
$$

When we truncate the matrices, we find that

$$
A_{N}(f) \neq \prod_{p} A_{N}\left(f_{p}\right) .
$$

However, even though this formula breaks down, it is miraculously recovered by taking determinants. Thus we shall prove that

$$
\operatorname{det} A_{N}(f)=\prod_{p} \operatorname{det} A_{N}\left(f_{p}\right)
$$

This reduces the problem to the evaluation of $\operatorname{det} A_{N}\left(f_{p}\right)$ for a given prime $p$. Define $\widehat{f}_{p}: \mathbb{T} \rightarrow \mathbb{T}$ (where $\mathbb{T}$ is the unit circle) by

$$
\widehat{f}_{p}\left(e^{i \theta}\right)=f_{p}\left(\frac{\theta}{\log p}\right) \quad(0 \leq \theta<2 \pi) .
$$

Write $c_{k}$ for $c\left(p^{k}\right)$. The matrix $A\left(f_{p}\right)$ contains only entries $(i, j)$ where $i / j$ is a power of $p$; the $(i, j)$ th entry is $c_{k}$ if $i / j=p^{k}$. For instance for $p=2$ it looks like 


$$
A\left(f_{2}\right)=\left(\begin{array}{ccccccccc}
c_{0} & c_{-1} & 0 & c_{-2} & 0 & 0 & 0 & c_{-3} & \cdots \\
c_{1} & c_{0} & 0 & c_{-1} & 0 & 0 & 0 & c_{-2} & \cdots \\
0 & 0 & c_{0} & 0 & 0 & c_{-1} & 0 & 0 & \cdots \\
c_{2} & c_{1} & 0 & c_{0} & 0 & 0 & 0 & c_{-1} & \cdots \\
0 & 0 & 0 & 0 & c_{0} & 0 & 0 & 0 & \cdots \\
0 & 0 & c_{1} & 0 & 0 & c_{0} & 0 & 0 & \cdots \\
0 & 0 & 0 & 0 & 0 & 0 & c_{0} & 0 & \cdots \\
c_{3} & c_{2} & 0 & c_{1} & 0 & 0 & 0 & c_{0} & \cdots \\
\vdots & \vdots & \vdots & \vdots & \vdots & \vdots & \vdots & \vdots & \ddots
\end{array}\right)
$$

For $a \in L^{\infty}(\mathbb{T})$, we denote (as is usual) by $T(a)$ the Toeplitz matrix with symbol $a$, and by $T_{N}(a)$ its $N \times N$-truncation. Thus

$$
T\left(\widehat{f}_{p}\right)=\left(\begin{array}{ccccc}
c_{0} & c_{-1} & c_{-2} & c_{-3} & \cdots \\
c_{1} & c_{0} & c_{-1} & c_{-2} & \cdots \\
c_{2} & c_{1} & c_{0} & c_{-1} & \cdots \\
c_{3} & c_{2} & c_{1} & c_{0} & \cdots \\
\vdots & \vdots & \vdots & \vdots & \ddots
\end{array}\right)
$$

For example, writing $c_{k}$ and $d_{k}$ for the coefficients of $f_{2}$ and $f_{3}$ respectively, we have

$$
\begin{aligned}
A_{4}\left(f_{2}\right) A_{4}\left(f_{3}\right) & =\left(\begin{array}{cccc}
c_{0} & c_{-1} & 0 & c_{-2} \\
c_{1} & c_{0} & 0 & c_{-1} \\
0 & 0 & c_{0} & 0 \\
c_{2} & c_{1} & 0 & c_{0}
\end{array}\right)\left(\begin{array}{cccc}
d_{0} & 0 & d_{-1} & 0 \\
0 & d_{0} & 0 & 0 \\
d_{1} & 0 & d_{0} & 0 \\
0 & 0 & 0 & d_{0}
\end{array}\right) \\
& =\left(\begin{array}{cccc}
c_{0} d_{0} & c_{-1} d_{0} & c_{0} d_{-1} & c_{-2} d_{0} \\
c_{1} d_{0} & c_{0} d_{0} & c_{1} d_{-1} & c_{-1} d_{0} \\
c_{0} d_{1} & 0 & c_{0} d_{0} & 0 \\
c_{2} d_{0} & c_{1} d_{0} & c_{2} d_{-1} & c_{0} d_{0}
\end{array}\right)
\end{aligned}
$$

while

$$
A_{4}\left(f_{2} f_{3}\right)=\left(\begin{array}{cccc}
c_{0} d_{0} & c_{-1} d_{0} & c_{0} d_{-1} & c_{-2} d_{0} \\
c_{1} d_{0} & c_{0} d_{0} & c_{1} d_{-1} & c_{-1} d_{0} \\
c_{0} d_{1} & c_{-1} d_{1} & c_{0} d_{0} & c_{-2} d_{1} \\
c_{2} d_{0} & c_{1} d_{0} & c_{2} d_{-1} & c_{0} d_{0}
\end{array}\right)
$$


So $A_{4}\left(f_{2}\right) A_{4}\left(f_{3}\right) \neq A_{4}\left(f_{2} f_{3}\right)$, but both the above matrices have determinant

$$
c_{0}\left|\begin{array}{ccc}
c_{0} & c_{-1} & c_{-2} \\
c_{1} & c_{0} & c_{-1} \\
c_{2} & c_{1} & c_{0}
\end{array}\right| d_{0}^{2}\left|\begin{array}{cc}
d_{0} & d_{-1} \\
d_{1} & d_{0}
\end{array}\right|
$$

Our main results are the following:

TheORem 3.1. Let $p$ be a prime number. Then

$$
\operatorname{det} A_{N}\left(f_{p}\right)=\prod_{r \geq 1}\left(\operatorname{det} T_{r}\left(\widehat{f}_{p}\right){ }^{\left[N / p^{r-1}\right]-2\left[N / p^{r}\right]+\left[N / p^{r+1}\right]} .\right.
$$

(The product is of course finite with $r \leq \frac{\log N}{\log p}+1$.)

TheOREM 3.2. Let $f \in U$ and suppose $f$ has multiplicative $\mathbb{Q}^{+}$-coefficients. Then

$$
\operatorname{det} A_{N}(f)=\prod_{p} \operatorname{det} A_{N}\left(f_{p}\right)=\prod_{p} \prod_{r \geq 1}\left(\operatorname{det} T_{r}\left(\widehat{f}_{p}\right)\right)^{\left[N / p^{r-1}\right]-2\left[N / p^{r}\right]+\left[N / p^{r+1}\right]} .
$$

REMARKS 3.3. The restriction of $f$ to $U$ is clearly unnecessary (in both Theorems 3.1 and 3.2) since the results only concern the values $c(q)$ with $|q| \leq N$. The only necessary assumption is that $c(\cdot)$ is multiplicative on $\left\{q \in \mathbb{Q}^{+}:|q| \leq N\right\}$. We retain the notation $A(f)$ since the form of $f$ (and hence of $\left.\widehat{f}_{p}\right)$ is useful in evaluating $\operatorname{det} T_{r}\left(\widehat{f}_{p}\right)$.

On noticing that the exponent of $\operatorname{det} T_{r}\left(\widehat{f}_{p}\right)$ is positive for $r \leq\left[\frac{\log N}{\log p}\right]-1$, we have the following corollary:

Corollary 3.4.

(i) The matrix $A_{N}(f)$ is invertible if and only if $A_{N}\left(f_{p}\right)$ is invertible for all $p$.

(ii) The matrix $A_{N}\left(f_{p}\right)$ is invertible for all $N$ if and only if $T_{r}\left(\widehat{f}_{p}\right)$ is invertible for all $r \geq 1$, in which case

$$
\operatorname{det} A_{N}\left(f_{p}\right)=\prod_{r \geq 1}\left(\frac{\left|T_{r-1}\left(\widehat{f}_{p}\right)\right|\left|T_{r+1}\left(\widehat{f}_{p}\right)\right|}{\left|T_{r}\left(\widehat{f}_{p}\right)\right|^{2}}\right)^{\left[N / p^{r}\right]},
$$

where $\left|T_{r}\left(\widehat{f}_{p}\right)\right|=\operatorname{det} T_{r}\left(\widehat{f}_{p}\right)$ and $\left|T_{0}\left(\widehat{f}_{p}\right)\right|=1$. Hence, if $T_{r}\left(\widehat{f}_{p}\right)$ is invertible for all $r \geq 1$ and all primes $p$, we have

$$
\operatorname{det} A_{N}(f)=\prod_{p} \prod_{r \geq 1}\left(\frac{\left|T_{r-1}\left(\widehat{f}_{p}\right)\right|\left|T_{r+1}\left(\widehat{f}_{p}\right)\right|}{\left|T_{r}\left(\widehat{f}_{p}\right)\right|^{2}}\right)^{\left[N / p^{r}\right]} .
$$


4. Proofs of Theorems 3.1 and 3.2. We start with some preliminaries.

Definition 4.1. Let $n>1$ be squarefree, say $n=p_{1} \cdots p_{k}$. Define sets $S_{n}$ and $S_{n}^{+}$by $\left(^{1}\right)$

$$
\begin{aligned}
S_{n} & =\left\{p_{1}^{a_{1}} \cdots p_{k}^{a_{k}}: a_{1}, \ldots, a_{k} \in \mathbb{Z}\right\}, \\
S_{n}^{+} & =\left\{p_{1}^{a_{1}} \cdots p_{k}^{a_{k}}: a_{1}, \ldots, a_{k} \geq 0\right\}=S_{n} \cap \mathbb{N} .
\end{aligned}
$$

It is straightforward to prove the following basic properties:

(i) $S_{n}$ is a group under multiplication;

(ii) if $d \mid n$, then $S_{d}^{+} \subset S_{n}^{+}$;

(iii) if $k \in S_{n}^{+}$and $d \mid k$, then $d \in S_{n}^{+}$.

Definition 4.2. Define a relation $\sim_{n}$ on a subset $T$ of $\mathbb{N}$ as follows: for $i, j \in T$,

$$
i \sim_{n} j \Leftrightarrow i / j \in S_{n} .
$$

That $\sim_{n}$ is an equivalence relation follows immediately from the fact that $S_{n}$ is a group under multiplication.

Lemma 4.3. Let $T$ be a subset of $\mathbb{N}$ which satisfies the condition: if $m \in T$, then $d \in T$ for every divisor $d$ of $m$. Then the equivalence classes of the relation $\sim_{n}$ on $T$ are given by

$[k]=k S_{n}^{+} \cap T=\left\{k s \in T: s \in S_{n}^{+}\right\} \quad$ for each $k \in T$ coprime to $n$.

Proof. For $k \in T$ such that $(k, n)=1$, define $[k]=k S_{n}^{+} \cap T$. Then $a, b \in[k]$ implies $a=k s$ and $b=k t$ for some $s, t \in S_{n}^{+}$. Hence $a / b=s / t \in S_{n}$, and so $a \sim_{n} b$. Next, every $j \in T$ is in some $[k]$. For we can write $j=\prod_{p} p^{\alpha_{p}}$ for some $\alpha_{p}$, so that

$$
j=\prod_{p \mid n} p^{\alpha_{p}} \prod_{p \nmid n} p^{\alpha_{p}}=s k,
$$

where $s=\prod_{p \mid n} p^{\alpha_{p}} \in S_{n}^{+}$and $k$ is coprime to $n$, and $k \in T$ since $k \mid j$. Hence $j \in[k]$.

In particular, we can take $T=T_{N}:=\{1, \ldots, N\}$. Further, by property (iii) above, we can (and shall) take $T=S_{m}^{+} \cap T_{N}$ for some $m$. We shall sometimes write $[k]_{n}$ or $[k]_{n, T}$ to denote the equivalence classes, to emphasise the dependence on $n$ and the set $T$.

Define the counting function

$$
S_{n}(x)=\sum_{\substack{s \leq x \\ s \in S_{n}^{+}}} 1 .
$$

$\left.{ }^{1}\right)$ The definition of $S_{n}$ was made earlier in $\S 2$, but we include it here for convenience. 
Thus $\left|[k]_{n, T_{N}}\right|=S_{n}(N / k)$, and since $\sim_{n}$ partitions $T_{N}$, we have

$$
\sum_{\substack{1 \leq k \leq N \\(k, n)=1}} S_{n}(N / k)=\left|T_{N}\right|=N .
$$

Slightly more generally, using the fact that $[x / k]=[[x] / k]$, we have, for $x \geq 0$,

$$
\sum_{\substack{k \leq x \\(k, n)=1}} S_{n}(x / k)=[x] .
$$

We shall write

$$
S_{n}^{+}=\left\{s_{1}^{(n)}, s_{2}^{(n)}, \ldots\right\} \quad \text { where } \quad 1=s_{1}^{(n)}<s_{2}^{(n)}<\cdots .
$$

Proof of Theorem 3.1. Since $p$ is prime, the equivalence relation $\sim_{p}$ on $T_{N}=\{1, \ldots, N\}$ is now: for $i, j \in T_{N}$,

$$
i \sim_{p} j \Leftrightarrow i / j=p^{m} \text { for some integer } m .
$$

The equivalence classes are given by

$$
[k]=\left\{k p^{m}: m \geq 0\right\} \cap T_{N}, \quad \text { where } k \in T_{N} \text { and } p \nmid k .
$$

The $(i, j)$ th entry of the matrix $A_{N}\left(f_{p}\right)$ is $c_{f_{p}}(i / j)$. This entry is zero if $i \varkappa_{p} j$, while if $i \sim_{p} j$, say $i / j=p^{m}$, then it is $c\left(p^{m}\right)=c_{m}$.

Consider the first equivalence class $[1]=\left\{1, p, p^{2}, \ldots, p^{r-1}\right\}$ for some $r$. Swap the rows labelled $1, p, \ldots, p^{r-1}$ with the first $r$ rows, then swap the columns labelled $1, p, \ldots, p^{r-1}$ with the first $r$ columns. The determinant remains the same $\left({ }^{2}\right)$, but now the top left $r \times r$-matrix has entries $a_{i j}=$ $c_{f_{p}}\left(p^{i-1} / p^{j-1}\right)=c_{i-j}$, i.e. it is the Toeplitz matrix $T_{r}\left(\widehat{f}_{p}\right)$. Furthermore, all remaining entries in the first $r$ rows and columns are zero. Now apply the same procedure for the next equivalence class, say $\left[k_{1}\right]=\left\{k_{1}, k_{1} p, \ldots, k_{1} p^{s-1}\right\}$ for some $s$. Swap rows and columns labelled $\left({ }^{3}\right) k_{1}, k_{1} p, \ldots, k_{1} p^{s-1}$ with the $s$ rows and columns directly after the first $r$. Repeating this for each equivalence class and rearranging the matrix accordingly results in a matrix with blocks down the diagonal and zeros elsewhere; that is,

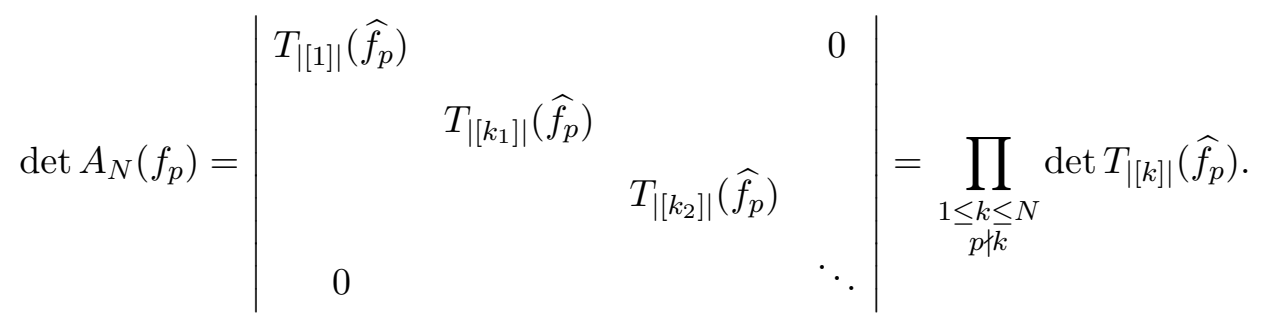

$\left({ }^{2}\right)$ The determinant of a matrix remains unchanged after swapping rows $k$ and $l$ and columns $k$ and $l$.

$\left({ }^{3}\right)$ Using the labelling of the original matrix. 
The size of a class $[k]$ is

$$
|[k]|=\sum_{m \geq 0, k p^{m} \leq N} 1=\sum_{0 \leq m \leq \frac{\log N / k}{\log p}} 1=\left[\frac{\log N / k}{\log p}\right]+1 .
$$

We require the number of equivalence classes of a given size $r$; that is, for how many $k \in T_{N}$ with $p \nmid k$ is

$$
r=\left[\frac{\log N / k}{\log p}\right]+1 ?
$$

This holds if and only if $N / p^{r}<k \leq N / p^{r-1}$, and so the required number is

$$
\sum_{\substack{N / p^{r}<k \leq N / p^{r-1} \\ p \nmid k}} 1=\sum_{N / p^{r}<k \leq N / p^{r-1}} 1-\sum_{N / p^{r+1}<k \leq N / p^{r}} 1=\left[\frac{N}{p^{r-1}}\right]-2\left[\frac{N}{p^{r}}\right]+\left[\frac{N}{p^{r+1}}\right] .
$$

Hence

$$
\operatorname{det} A_{N}\left(f_{p}\right)=\prod_{1 \leq r \leq[\log N / \log p]+1}\left(\operatorname{det} T_{r}\left(\widehat{f}_{p}\right)\right)^{\left[N / p^{r-1}\right]-2\left[N / p^{r}\right]+\left[N / p^{r+1}\right]} .
$$

For the proof of Theorem 3.2, we first need a preliminary result, regarding the determinant of a certain type of block matrix. For a matrix $A=\left(a_{i j}\right)$, denote by $A_{m, n}$ the $m \times n$-truncation of $A$; that is, $A_{m, n}=\left(a_{i j}\right)_{i \leq m, j \leq n}$ $(m, n \in \mathbb{N})$. Further, write $A_{n}$ for $A_{n, n}$.

Proposition 4.4. Let $l_{1}, \ldots, l_{n}$ be positive integers such that $l_{1} \geq \cdots \geq$ $l_{n} \geq 1$. Let $B=\left(b_{i j}\right)$ and $C=\left(c_{i j}\right)$ be arbitrary matrices. Let $A$ be the matrix formed by the blocks $\left(b_{r s} C_{l_{r}, l_{s}}\right)_{r, s \leq n}$; that is, A equals

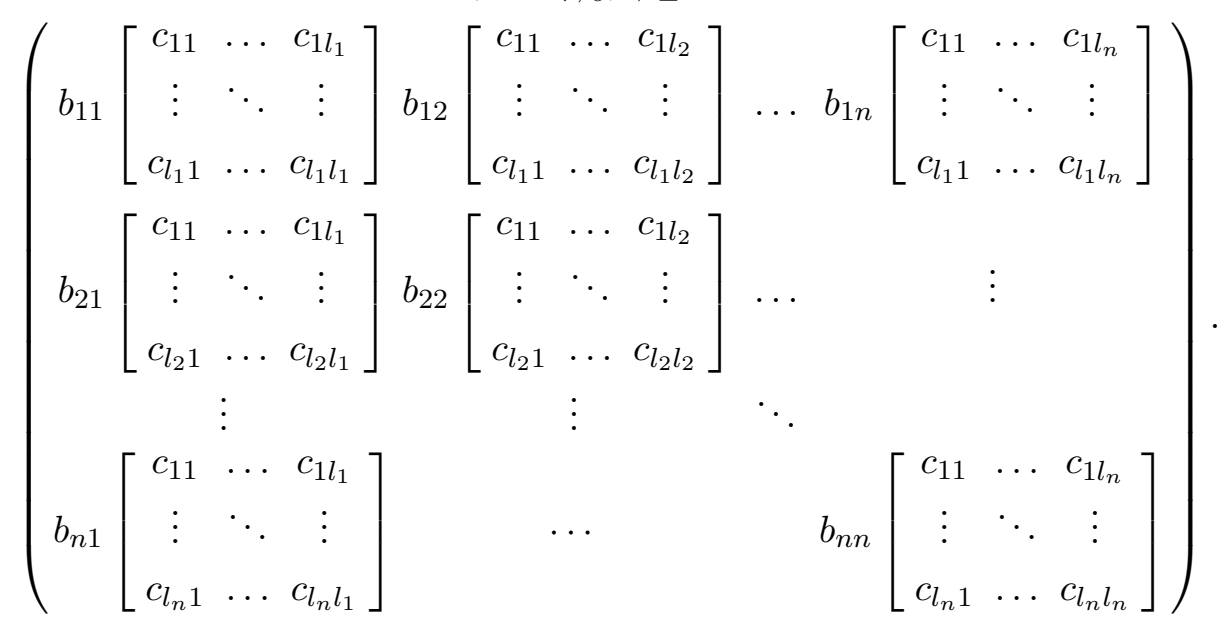

Then

where $l_{n+1}=0$.

$$
\operatorname{det} A=\prod_{m=1}^{n}\left(\operatorname{det} B_{m}\right)^{l_{m}-l_{m+1}}\left(\operatorname{det} C_{l_{m}}\right)
$$


Proof. We can describe $A=\left(a_{i j}\right)$ as follows: put $L_{0}=0$ and $L_{r}=$ $l_{1}+\cdots+l_{r}$ for $r=1, \ldots, n$. Then for $L_{r-1}<i \leq L_{r}$ and $L_{s-1}<j \leq L_{s}$,

$$
a_{i j}=b_{r s} c_{i-L_{r-1}, j-L_{s-1}} .
$$

We call the submatrix where $L_{r-1}<i \leq L_{r}$ and $L_{s-1}<j \leq L_{s}$ the block $(r, s)$.

We prove the result by induction on $n$. If $n=1$, we have $\left|b_{11} C_{l_{1}}\right|=$ $b_{11}^{l_{1}}\left|C_{l_{1}}\right|=\left|B_{1}\right|^{l_{1}}\left|C_{l_{1}}\right|$, as required. Now assume the result holds for all such matrices with $k \leq n-1$ blocks down the diagonal. Since determinants vary continuously with respect to the entries, it suffices to prove the result when $b_{11} \neq 0$, which we now assume.

Apply the first step in Gaussian elimination: subtract suitable multiples of the first number of rows from each of the blocks in the first column to obtain zeros below the leading block; that is, for $L_{r-1}<i \leq L_{r}$ and $r=2, \ldots, n$, we apply the rule

$$
r_{i} \mapsto r_{i}-\frac{b_{r 1}}{b_{11}} r_{i-L_{r-1}} .
$$

Then the $(r, s)$-block $L_{r-1}<i \leq L_{r}, L_{s-1}<j \leq L_{s}$ transforms as

$a_{i j} \rightarrow a_{i j}-\frac{b_{r 1}}{b_{11}} a_{i-L_{r-1}, j}=\left(b_{r s}-\frac{b_{r 1} b_{1 s}}{b_{11}}\right) c_{i-L_{r-1}, j-L_{s-1}}=b_{r s}^{\prime} c_{i-L_{r-1}, j-L_{s-1}}$,

say, where $b_{r s}^{\prime}=b_{r s}-b_{r 1} b_{1 s} / b_{11}$. Here we used the fact that $L_{r-1}<i \leq L_{r}$ implies $0<i-L_{r-1} \leq l_{r} \leq l_{1}$, so that $a_{i-L_{r-1}, j}$ lies in block $(1, s)$. Note that $b_{r 1}^{\prime}=0$ so the first column of blocks (apart from the top one) are now all zero. Hence

$$
\begin{aligned}
|A| & =\left|\left(b_{r s} C_{l_{r}, l_{s}}\right)_{r, s \leq n}\right|=\left|\begin{array}{cc}
b_{11} C_{l_{1}} & * \\
0 & \left(b_{r s}^{\prime} C_{l_{r}, l_{s}}\right)_{2 \leq r, s \leq n}
\end{array}\right| \\
& =b_{11}^{l_{1}}\left|C_{l_{1}}\right|\left|\left(b_{r s}^{\prime} C_{l_{r}, l_{s}}\right)_{2 \leq r, s \leq n}\right| .
\end{aligned}
$$

On the right we again have a determinant of a "block" matrix, but this time with $n-1$ blocks along the diagonal. By inductive hypothesis, we have

$$
|A|=\left|B_{1}\right|^{l_{1}}\left|C_{l_{1}}\right| \cdot\left|B_{1}^{\prime}\right|^{l_{2}-l_{3}} \cdots\left|B_{n-1}^{\prime}\right|^{l_{n}-l_{n+1}}\left|C_{l_{2}}\right| \cdots\left|C_{l_{n}}\right|,
$$

where $B^{\prime}=\left(b_{i j}^{\prime}\right)_{i, j \geq 2}$. But applying the same first step of Gaussian elimination to $B_{r}$ itself, we get

$$
\left|B_{r}\right|=\left|\begin{array}{cc}
b_{11} & * \\
0 & B_{r-1}^{\prime}
\end{array}\right|=b_{11}\left|B_{r-1}^{\prime}\right| .
$$


Hence $\left|B_{r-1}^{\prime}\right|=\left|B_{r}\right| /\left|B_{1}\right|$, and (4.2) becomes

$$
\begin{aligned}
|A| & =\left|B_{1}\right|^{l_{1}} \frac{\left|B_{2}\right|^{l_{2}-l_{3}} \cdots\left|B_{n}\right|^{l_{n}-l_{n+1}}}{\left|B_{1}\right|^{l_{2}-l_{3}} \cdots\left|B_{1}\right|^{l_{n}-l_{n+1}}}\left|C_{l_{1}}\right| \cdots\left|C_{l_{n}}\right| \\
& =\left|B_{1}\right|^{l_{1}-l_{2}}\left|B_{2}\right|^{l_{2}-l_{3}} \ldots\left|B_{n}\right|^{l_{n}-l_{n+1}}\left|C_{l_{1}}\right| \cdots\left|C_{l_{n}}\right| .
\end{aligned}
$$

Proof of Theorem 3.2. Note that $\operatorname{det} A_{N}(f)=\operatorname{det} A_{N}\left(f_{n}\right)$ where $n=$ $\prod_{p \mid N} p$, since the coefficients $c(i / j)$ with $i$ or $j$ greater than $N$ do not affect the matrix $A_{N}(f)$. Indeed, we shall evaluate $\operatorname{det} A_{N}\left(f_{n}\right)$ for any $n$ squarefree and any $N$ by proving

$$
\operatorname{det} A_{N}\left(f_{n}\right)=\prod_{p \mid n} \operatorname{det} A_{N}\left(f_{p}\right) .
$$

If $n$ is prime, then there is nothing more to prove. Suppose $n$ has at least two prime factors. We can write $n=m p$ where $p$ is prime and $p \nmid m$. Then the above follows if we can prove that

$$
\operatorname{det} A_{N}\left(f_{n}\right)=\operatorname{det} A_{N}\left(f_{p}\right) \operatorname{det} A_{N}\left(f_{m}\right) .
$$

We begin as in the proof of Theorem 3.1, this time swapping the rows and columns of $A_{N}\left(f_{n}\right)$ corresponding to each of the equivalence classes of $\sim_{n}$ on the set $T_{N}$ in turn. They are

$$
[k]=[k]_{n, T_{N}}=\left\{k s_{1}^{(n)}, k s_{2}^{(n)}, \ldots\right\}, \quad \text { where }(k, n)=1 \text { and } k \leq N .
$$

Swap rows and columns labelled by the elements of [1] with the first $|[1]|$ rows and columns. The top left $|[1]| \times|[1]|$-matrix now has entries $b_{i j}=$ $c\left(s_{i}^{(n)} / s_{j}^{(n)}\right)$. Then swap the rows and columns labelled by the elements of the next equivalence class, say $\left[k_{1}\right]$, with the next $\left|\left[k_{1}\right]\right|$ rows and columns. Repeat this procedure for all the equivalence classes. As in the case $n=p$ of Theorem 3.1, we obtain a matrix with blocks down the diagonal and zeros elsewhere:

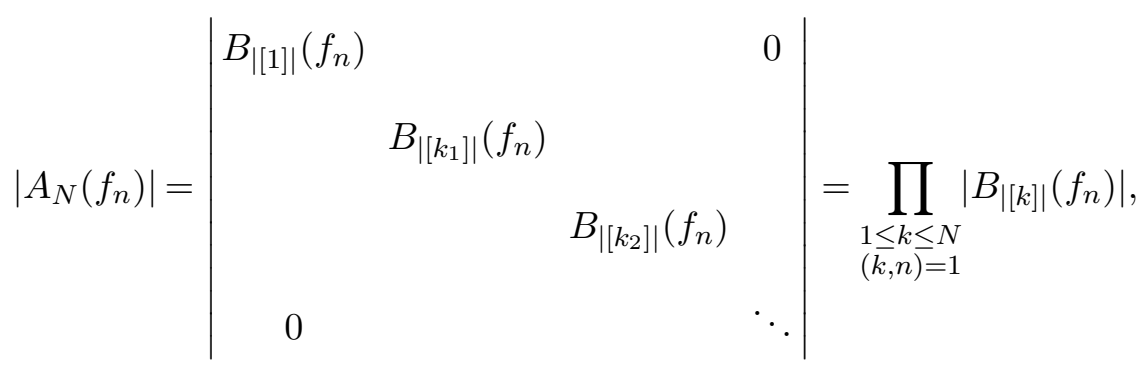

where $B\left(f_{n}\right)=\left(b_{i j}\right)$ with $b_{i j}=c\left(s_{i}^{(n)} / s_{j}^{(n)}\right)$, and $B_{r}\left(f_{n}\right)$ is the $r \times r$ truncation of $B\left(f_{n}\right)$. 
Let $k \in T_{N}$ be such that $(k, n)=1$. Define the set $T^{(k)}$ by

$$
T^{(k)}:=[1]_{n, T_{M}}=S_{n}^{+} \cap T_{M}, \quad \text { where } M=[N / k],
$$

and let $t_{k}$ denote its cardinality, i.e.

$$
t_{k}=\left|T^{(k)}\right|=S_{n}(N / k) .
$$

Note that $\left|[k]_{n, T_{N}}\right|=\left|[1]_{n, T_{M}}\right|=t_{k}$, so that $B_{\left|[k]_{n, T_{N}}\right|}\left(f_{n}\right)=B_{t_{k}}\left(f_{n}\right)$, and (4.4) becomes

$$
\left|A_{N}\left(f_{n}\right)\right|=\prod_{\substack{1 \leq k \leq N \\(k, n)=1}}\left|B_{t_{k}}\left(f_{n}\right)\right| .
$$

Consider the equivalence relation $\sim_{m}$ on $T^{(k)}$. By Lemma 4.3, the equivalence classes are

$$
[j]_{m, T}=j S_{m}^{+} \cap T^{(k)}=j S_{m}^{+} \cap S_{n}^{+} \cap T_{M}=j S_{m}^{+} \cap T_{M}=[j]_{m, T_{M}}
$$

(since $j S_{m}^{+} \subset S_{n}^{+}$) for $j \in T$ such that $(j, m)=1$, i.e. $j \in S_{n}^{+}$and $j \leq M$ and $(j, m)=1$. But $j \in S_{n}^{+}$implies $j=p^{r} j^{\prime}$ for some $r \geq 0$ and $j^{\prime} \in S_{m}^{+}$. Since $j$ is coprime to $m$, we must have $j^{\prime}=1$, hence $j$ is a power of $p$ only. Thus the equivalence classes are

$$
\left[p^{r}\right]_{m, T_{M}}=\left\{p^{r} s_{1}^{(m)}, p^{r} s_{2}^{(m)}, \ldots, p^{r} s_{l}^{(m)}\right\} \quad \text { (for some } l \text { ) }
$$

for $r=0,1, \ldots$ such that $p^{r} \leq M$. Note that $\left|\left[p^{r}\right]_{m, T_{M}}\right|=S_{m}\left(N / k p^{r}\right)$.

Label the rows and columns of each matrix $B_{t_{k}}\left(f_{n}\right)$ by the elements of $T^{(k)}$. Now swap the rows and columns of each $B_{t_{k}}\left(f_{n}\right)$ according to the equivalence classes of $\sim_{m}$ on $T^{(k)}$. That is, swap the rows and columns labelled by the elements of $[1]_{m, T_{M}}$ with the first $\left|[1]_{m, T_{M}}\right|$ rows and columns. Then swap those labelled by $[p]_{m, T_{M}}$ with the next $\left|[p]_{m, T_{M}}\right|$ rows and columns. Repeat this for each of the equivalence classes. The resulting determinant now has its rows and columns ordered as

$$
[1]_{m},[p]_{m},\left[p^{2}\right]_{m}, \ldots
$$

For this new determinant, the $(i, j)$ th entry in the "block" corresponding to the equivalence classes $\left[p^{r-1}\right]_{m}$ and $\left[p^{s-1}\right]_{m}$ is

$$
\begin{aligned}
c\left(\frac{p^{r} s_{i}^{(m)}}{p^{s} s_{j}^{(m)}}\right) & \left.=c\left(p^{r-s}\right) c\left(\frac{s_{i}^{(m)}}{s_{j}^{(m)}}\right) \quad \text { (by multiplicativity of } c(\cdot)\right) \\
& =c_{r-s} B\left(f_{m}\right)_{i j} .
\end{aligned}
$$

The resulting matrix is therefore in the block form as in Proposition 4.4, with " $B "=T\left(\widehat{f}_{p}\right), " C$ " $=B\left(f_{m}\right)$, and

$$
\text { "l } l_{r}=l_{r, k}:=\left|\left[p^{r-1}\right]_{m, T_{M}}\right|=S_{m}\left(N / k p^{r-1}\right) .
$$


By Proposition 4.4, we have

$$
\left|B_{t_{k}}\left(f_{n}\right)\right|=\prod_{r \geq 1}\left|T_{r}\left(\widehat{f}_{p}\right)\right|^{l_{r, k}-l_{r+1, k}}\left|B_{l_{r, k}}\left(f_{m}\right)\right| .
$$

(This is a finite product since the $l_{r, k}$ are eventually zero. Indeed, $l_{r, k}=0$ when $k p^{r-1}>N$.) Substituting into (4.5) gives

$$
\begin{aligned}
\left|A_{N}\left(f_{n}\right)\right| & =\prod_{\substack{1 \leq k \leq N \\
(k, n)=1}} \prod_{r \geq 1}\left|T_{r}\left(\widehat{f}_{p}\right)\right|^{l_{r, k}-l_{r+1, k}}\left|B_{l_{r, k}}\left(f_{m}\right)\right| \\
& =\left(\prod_{r \geq 1}\left|T_{r}\left(\widehat{f}_{p}\right)\right|^{e_{r}}\right)\left(\prod_{r \geq 1} \prod_{\substack{1 \leq k \leq N \\
(k, n)=1}}\left|B_{l_{r, k}}\left(f_{m}\right)\right|\right),
\end{aligned}
$$

where

$$
e_{r}=\sum_{\substack{1 \leq k \leq N \\(k, n)=1}}\left(l_{r, k}-l_{r+1, k}\right)=\sum_{\substack{1 \leq k \leq N \\(k, n)=1}}\left(S_{m}\left(N / k p^{r-1}\right)-S_{m}\left(N / k p^{r}\right)\right) .
$$

Now, $(k, n)=1$ if and only if $(k, m)=1$ and $p \nmid k$. Hence for $x \leq N$,

$$
\begin{aligned}
\sum_{\substack{1 \leq k \leq N \\
(k, n)=1}} S_{m}(x / k) & =\sum_{\substack{k \leq x \\
(k, m)=1, p \nmid k}} S_{m}(x / k)=\sum_{\substack{k \leq x \\
(k, m)=1}} S_{m}(x / k)-\sum_{\substack{p k \leq x \\
(k, m)=1}} S_{m}(x / p k) \\
& =[x]-[x / p] \quad \text { from }(4.1) .
\end{aligned}
$$

It follows that

$$
e_{r}=\left[\frac{N}{p^{r-1}}\right]-\left[\frac{N}{p^{r}}\right]-\left(\left[\frac{N}{p^{r}}\right]-\left[\frac{N}{p^{r+1}}\right]\right)=\left[\frac{N}{p^{r-1}}\right]-2\left[\frac{N}{p^{r}}\right]+\left[\frac{N}{p^{r+1}}\right] .
$$

Hence (4.6) implies (after Theorem 3.1)

$$
\left|A_{N}\left(f_{n}\right)\right|=\left|A_{N}\left(f_{p}\right)\right| \prod_{r \geq 1} \prod_{\substack{1 \leq k \leq N \\(k, n)=1}}\left|B_{l_{r, k}}\left(f_{m}\right)\right| .
$$

Furthermore, since $l_{r, k}=0$ when $k p^{r-1}>N$, we have

$$
\prod_{\substack{1 \leq k \leq N \\(k, n)=1}}\left|B_{l_{r, k}}\left(f_{m}\right)\right|=\frac{\prod_{\substack{k \leq N / p^{r-1} \\(k, m)=1}}\left|B_{l_{r, k}}\left(f_{m}\right)\right|}{\prod_{\substack{p k \leq N / p^{r-1} \\(k, m)=1}}\left|B_{l_{r, p k}}\left(f_{m}\right)\right|} .
$$


But $l_{r, p k}=l_{r+1, k}$, so

$$
\begin{aligned}
\prod_{r \geq 1} \prod_{\substack{1 \leq k \leq N \\
(k, n)=1}}\left|B_{l_{r, k}}\left(f_{m}\right)\right| & =\frac{\prod_{r \geq 1} \prod_{\substack{k \leq N / p^{r-1} \\
(k, m)=1}}\left|B_{l_{r, k}}\left(f_{m}\right)\right|}{\prod_{r \geq 2} \prod_{\substack{k \leq N / p^{r-1} \\
(k, m)=1}}\left|B_{l_{r, k}}\left(f_{m}\right)\right|} \\
& =\prod_{\substack{k \leq N \\
(k, m)=1}}\left|B_{l_{1, k}}\left(f_{m}\right)\right| \quad \text { (the } r=1 \text { term only) } \\
& =\left|A_{N}\left(f_{m}\right)\right| \quad \text { by }(4.5),
\end{aligned}
$$

since $l_{1, k}=t_{k}$. Thus (4.7) gives

$$
\left|A_{N}\left(f_{n}\right)\right|=\left|A_{N}\left(f_{p}\right)\right|\left|A_{N}\left(f_{m}\right)\right|,
$$

and the result follows.

5. Applications. We can use Theorem 3.2 to evaluate some interesting determinants. First suppose $f$ has completely multiplicative coefficients $c(q)$, such that $|c(p)|,|c(1 / p)|<1$. Then, for $t \in \mathbb{T}$,

$$
\begin{aligned}
\widehat{f}_{p}(t) & =\sum_{k=-\infty}^{\infty} c\left(p^{k}\right) t^{k}=\sum_{k=1}^{\infty} c(p)^{k} t^{k}+\sum_{k=0}^{\infty} c(1 / p)^{k} t^{-k} \\
& =\frac{c(p) t}{1-c(p) t}+\frac{t}{t-c(1 / p)}=\frac{(1-c(p) c(1 / p)) t}{(1-c(p) t)(t-c(1 / p))}
\end{aligned}
$$

This is a rational function, and explicit formulas for determinants of truncated Toeplitz matrices with rational symbols were found by Day (see, for example [1, p. 163]). Applying Day's formula, one finds that

Hence

$$
\operatorname{det} T_{n}\left(\widehat{f}_{p}\right)=(1-c(p) c(1 / p))^{n-1} \quad \text { for } n \geq 1 \text {. }
$$

$$
\frac{\left|T_{m-1}\left(\widehat{f}_{p}\right)\right|\left|T_{m+1}\left(\widehat{f}_{p}\right)\right|}{\left|T_{m}\left(\widehat{f}_{p}\right)\right|^{2}}= \begin{cases}1 & \text { if } m \geq 2 \\ 1-c(p) c(1 / p) & \text { if } m=1\end{cases}
$$

Corollary 3.4 gives

$$
\operatorname{det} A_{n}(f)=\prod_{p \leq n}(1-c(p) c(1 / p))^{[n / p]} .
$$

After Proposition 2.2, we can rewrite this more conveniently as follows: put $c(m / n)=f(m) g(n)$ whenever $(m, n)=1$ and where $f$ and $g$ are completely multiplicative functions on $\mathbb{N}$. Then

and we obtain:

$$
c\left(\frac{i}{j}\right)=c\left(\frac{i /(i, j)}{j /(i, j)}\right)=f\left(\frac{i}{(i, j)}\right) g\left(\frac{j}{(i, j)}\right)
$$


Corollary 5.1. Let $f, g: \mathbb{N} \rightarrow \mathbb{C}$ be completely multiplicative. Then

$$
\begin{aligned}
\operatorname{det}\left(f\left(\frac{i}{(i, j)}\right) g\left(\frac{j}{(i, j)}\right)\right)_{i, j \leq n} & =\prod_{p \leq n}(1-f(p) g(p))^{[n / p]} \\
& =\prod_{r=1}^{n} \prod_{p \mid r}(1-f(p) g(p)) .
\end{aligned}
$$

The last expression is a standard manipulation on the RHS.

For example, with $f(n)=1 / n^{\alpha}$ and $g(n)=1 / n^{\beta}(\alpha, \beta \in \mathbb{C})$, we obtain

$$
\operatorname{det}\left(\left(\frac{(i, j)^{\alpha+\beta}}{i^{\alpha} j^{\beta}}\right)_{i, j \leq n}\right)=\prod_{r=1}^{n} \prod_{p \mid r}\left(1-\frac{1}{p^{\alpha+\beta}}\right) .
$$

In particular, putting $\alpha=\beta=1 / 2$, we have

$$
\operatorname{det}\left(\left(\frac{(i, j)}{\sqrt{i j}}\right)_{i, j \leq n}\right)=\prod_{r=1}^{n} \frac{\phi(r)}{r} .
$$

More generally, taking $f(n)=h(n) / \sqrt{n}$ and $g(n)=1 /(\sqrt{n} h(n))$, with $h$ completely multiplicative and non-zero, Corollary 5.1 gives

$$
\operatorname{det}\left(\left(\frac{h(i)(i, j)}{h(j) \sqrt{i j}}\right)_{i, j \leq n}\right)=\prod_{r=1}^{n} \frac{\phi(r)}{r} .
$$

As a further example, let $a(n)=\mu(n) / n^{\sigma}$ and $b(n)$ completely multiplicative. Define $c(\cdot)$ as in Theorem 2.3. Then $c$ is multiplicative (but not completely). Moreover, by (2.1) (with $m=\prod_{p} p^{\alpha}, n=\prod_{p} p^{\beta}$ and $(m, n)=1$ ) we have

$$
\begin{aligned}
c\left(\frac{m}{n}\right) & =\prod_{p \mid m} \frac{a\left(p^{\alpha}\right)}{1+a(p) b(p)} \prod_{p \mid n} \frac{b\left(p^{\beta}\right)+a(p) b\left(p^{\beta+1}\right)}{1+a(p) b(p)} \\
& =\frac{a(m) b(n)}{\prod_{p \mid m}(1+a(p) b(p))} \quad \text { (since } b \text { is completely multiplicative) } \\
& =\frac{\mu(m) b(n)}{m^{\sigma}} \prod_{p \mid m} \frac{1}{1-b(p) p^{-\sigma}} .
\end{aligned}
$$

For $k \geq 1$ and $p$ prime, it follows that

$$
c\left(p^{k}\right)=\left\{\begin{array}{ll}
-1 /\left(p^{\sigma}-b(p)\right) & \text { if } k=1, \\
0 & \text { if } k \geq 2,
\end{array} \quad \text { and } \quad c\left(1 / p^{k}\right)=b(p)^{k} .\right.
$$

Hence

$$
\widehat{f}_{p}(t)=\sum_{k=-\infty}^{\infty} c\left(p^{k}\right) t^{k}=\frac{t\left(p^{\sigma}-t\right)}{\left(p^{\sigma}-b(p)\right)(t-b(p))}
$$


again a rational symbol. Applying Day's explicit formula for Toeplitz matrices with rational symbol gives

$$
\operatorname{det} T_{n}\left(\widehat{f}_{p}\right)=\left(1-b(p) / p^{\sigma}\right)^{1-n} \quad \text { for } n \geq 1 .
$$

Hence

$$
\frac{\left|T_{m-1}\left(\widehat{f}_{p}\right)\right|\left|T_{m+1}\left(\widehat{f}_{p}\right)\right|}{\left|T_{m}\left(\widehat{f}_{p}\right)\right|^{2}}= \begin{cases}1 & \text { if } m \geq 2 \\ 1 /\left(1-b(p) p^{-\sigma}\right) & \text { if } m=1 .\end{cases}
$$

Corollary 3.4 now gives

$$
\operatorname{det}(c(i / j))_{i, j \leq n}=\prod_{p \leq n}\left(1-b(p) / p^{\sigma}\right)^{-[n / p]} .
$$

As a special case take $b(n)=n^{\sigma-1}$. Then (5.2) gives for $m, n$ coprime

$$
c(m / n)=\frac{\mu(m) n^{\sigma-1}}{\phi(m) m^{\sigma-1}} .
$$

Hence

$$
\operatorname{det}\left(\frac{\mu(i /(i, j))}{\phi(i /(i, j))}\left(\frac{i}{j}\right)^{1-\sigma}\right)_{i, j \leq n}=\prod_{r=1}^{n} \frac{r}{\phi(r)} .
$$

\section{References}

[1] A. Böttcher and B. Silbermann, Introduction to Large Truncated Toeplitz Matrices, Springer, 1999.

[2] K. Bourque and S. Ligh, Matrices associated with classes of arithmetical functions, J. Number Theory 45 (1993), 367-376.

[3] P. Codecá and M. Nair, Calculating a determinant associated with multiplicative functions, Boll. Unione Mat. Ital. Sez. B Artic. Ric. Mat. (8) 5 (2002), 545-555.

[4] J. Hadamard, Théorème sur les séries entières, Acta Math. 22 (1899), 60-63.

[5] S. F. Hong, Gcd-closed sets and determinants of matrices associated with arithmetical functions, Acta Arith. 101 (2002), 321-332.

[6] P. McCarthy, A generalization of Smith's determinant, Canad. Math. Bull. 29 (1988), 109-113.

[7] H. J. S. Smith, On the value of a certain arithmetical determinant, Proc. London Math. Soc. 7 (1875-76), 208-212.

[8] O. Toeplitz, Zur Theorie der Dirichletschen Reihen, Amer. J. Math. 60 (1938), 880888.

Department of Mathematics

University of Reading Whiteknights

P.O. Box 220

Reading RG6 6AX, UK

E-mail: t.w.hilberdink@reading.ac.uk 